\title{
Surface Ultra-Structure and Size of Human Corneocytes from Upper Stratum Corneum Layers of Normal and Diabetic Subjects with Discussion of Cohesion Aspects
}

\author{
Markus Stücker ${ }^{1 *}$, Michael Licht ${ }^{2 \#}$ and H Michael Heise ${ }^{2}$ \\ ${ }^{1}$ Vein Center of the Departments of Dermatology and Vascular Surgery, Ruhr-University Bochum, D-44805 Bochum, Germany \\ ${ }^{2}$ Interdisciplinary Center for Life Sciences, University of Applied Sciences of South-Westphalia, D-58644 Iserlohn, Germany
}

\begin{abstract}
Background: During the final stage of differentiation of cornified squamous epithelia like the human skin epidermis, anucleated corneocytes are formed. Formation of the horny layer and its ongoing desquamation are fundamental processes leading to the formation of an efficient epidermal barrier.

Materials and methods: For a better understanding of the desquamation process, the role of corneocyte surface ultra-structure has been investigated using a special preparation technique for scanning electron microscopy (SEM). Human morphologically different corneocytes from the stratum corneum of the fingertip, the thenar eminence (thick skin), and the wrist below the carpus (thin skin) of normal and diabetic subjects were obtained by adhesive tape stripping.

Results: The inside surface structure of corneocytes from thick skin shows prominent nubs, which are broader and more extended than those of thin skin. Towards their outside, corneocytes were flat with cavities as indentations of the nubs from neighboured cells providing clues on the mechanical strength of the 'intercellular stickiness'. As the size of thin skin corneocytes for diabetic subjects was also studied, it was found that their area was slightly nonlinearly dependent on age.

Conclusion: Accordingly to a reduced proliferation and differentiation rate, as postulated for diabetic persons, differences in size were as expected but statistically not significant, compared with corneocytes under normal homeostasis conditions. For discussion is a model, for which the interwoven cellular connectivity provides additional mechanical strength for the stratum corneum in thick skin.
\end{abstract}

Keywords: Human skin; Scanning electron microscopy; Stratum corneum; Ultrastructure; Corneocytes; Cellular cohesion

\section{Introduction}

The stratum corneum (SC) is composed of keratinocytes at different stages, joined together with corneodesmosomes, embedded in a hydrophobic lipid matrix. During the final stage of differentiation of cornified squamous epithelia, such as the skin epidermis, anucleated corneocytes are formed. Formation of the horny layer and its ongoing desquamation, i.e. the shedding of superficial corneocytes from the skin surface, are fundamental processes leading to the establishment of an efficient epidermal barrier. In the past, cohesion and desquamation of cells within the upper stratum corneum layers have often been studied allowing us to understand its formation and homeostasis [1-3].

The SC integrity as discussed in earlier publications mainly depends on three elements: 1) the physico-chemical quality of the cornified cells, 2) the persistence of mechanical junctions (corneodesmosomes) connecting these cells and 3) the organization and composition of the lipid "mortar" in the intercellular spaces. The stratum corneum can be shedded into single squames in different ways; these include mechanical desquamation as well as the use of agents such as detergents and enzymes.

In the lower SC, called stratum corneum compactum, corneocytes are tightly superimposed and attached by several corneodesmosomes (modified stratum corneum desmosomes). Further towards the SC surface, corneodesmosomes located in the central parts of the corneocyte disks start to disappear rapidly and the cells detach at these regions, forming a much loosely SC structure - the stratum corneum disjunctum. Corneocyte desquamation follows the degradation of the cell-cell junctions, including the lateral corneodesmosomes. The desquamation process is a precisely-controlled cascade of proteolytic events. Ultimately, the breakdown of the intracellular connections allows normal desquamation [4]. Note that the corneosomal structure represents the primary cohesive force, which must be degraded for desquamation according to Harding [5].

Ultrastructural aspects using electron microscopic methods for investigating the stratum corneum have been reviewed by Haftek [6]. Such studies have been previously carried out on various occasions using scanning electron microscopy (SEM) [7-9]. Despite the demands for highest quality, usually electron microscopic techniques show some deficiencies for the characterisation of the corneocyte surface structures [10]. Native corneocytes are found in hydrated form, but for transmission electron microscopy (TEM) a previous exposure to organic solvents and the vacuum implicate morphological changes

*Corresponding author: Prof. Dr. Markus Stücker, Vein Center of the Departments of Dermatology and Vascular Surgery, Ruhr-University Bochum, D-44805 Bochum Germany, Tel: 00492348792 377; E-mail: m.stuecker@klinikum-bochum.de

Received April 15, 2015; Accepted September 11, 2015; Published September 15, 2015

Citation: Stücker M, Licht M, Heise HM (2015) Surface Ultra-Structure and Size of Human Corneocytes from Upper Stratum Corneum Layers of Normal and Diabetic Subjects with Discussion of Cohesion Aspects. J Diabetes Metab 6: 603. doi:10.4172/2155-6156.1000603

Copyright: $\odot 2015$ Stücker M, et al. This is an open-access article distributed unde the terms of the Creative Commons Attribution License, which permits unrestricted use, distribution, and reproduction in any medium, provided the original author and source are credited. 
Citation: Stücker M, Licht M, Heise HM (2015) Surface Ultra-Structure and Size of Human Corneocytes from Upper Stratum Corneum Layers of Normal and Diabetic Subjects with Discussion of Cohesion Aspects. J Diabetes Metab 6: 603. doi:10.4172/2155-6156.1000603

Page 2 of 5

[11]. Often the SEM studies show the stratum corneum as a crosssection of the tissue with gaps between the corneocytes. With SEM freeze-fracture measurements, the three-dimensional structure of the squamous epithelium cells is only partially elucidated as detailed by Warner et al. [12].

For preparing SC samples for SEM, skin surface biopsy techniques are used. For collecting integral SC layers, a cyanoacrylate adhesive can also be employed for detaching such material, as described by Plewig et al. [10] and Marks [13]. However, for our investigations the adhesive tape stripping method was applied, which allows to study isolated corneocytes. The tape stripping method has been described in detail by Lindemann et al. [14] with a particular view to the density of corneocytes on the adhesive tape and their quantitative assessment. For chemical penetration and bioavailability studies, skin surface stripping with adhesive tapes has been used within dermatology, pharmacology, and cosmetics (skin care) on various occasions. With multiple strippings, even a complete removal of the stratum corneum of the epidermis can be achieved [15,16].

An early extensive SEM study on the structure and organisation of human stratum corneum was carried out by Menton and Eisen [9], who looked at horny cells, e.g. from palm and limb surfaces, focussing much on the cornecyte overlapped and interdigitated junctions. They observed a villous structure of cells from thick skin with their surface pointing towards the dermis, but did not study the counterpart surface. Here, by means of a simple procedure using a conductive adhesive tape, SEM measurements of the dehydrated corneocytes surface, towards the in- and outside of the body, were carried out successfully for thick and thin skin samples, showing significant morphological differences in their ultra-structures [17-23]. Ex vivo corneocyte structures were investigated in order to better understand also the mechanisms that regulate desquamation at the epidermal surface, as well as mechanical stability of the stratum corneum as studied shortly before by Wu et al. [24].

\section{Materials and Methods}

The skin below the wrist of six subjects of age between 25 and 84, without any skin diseases known, was studied. After cleansing the skin surface with ethanol and a short elapsed time required for ethanol evaporation, adhesive tape (Tesa No. 4204, Beiersdorf AG, Hamburg, Germany) was pressed onto the skin and torn away. The tape stripping procedure was applied twice. The tapes were stored on a clean microscope slide. For two subjects, the same procedure was carried out for the thick skin of the fingertip and that of the heel of hand. The study had been approved by the ethics committee of the medical faculty of the Ruhr-University, Bochum, Germany. Informed consent was obtained from all subjects $(\mathrm{N}=17)$ before the study.

The adhering corneocytes, single or as an ensemble of cells, were sticking to the tape with their surfaces that were pointing towards the body outside. Part of the tape was transferred with the non-adhesive side to a Leit-Tab tape (Plano Wetzlar Germany), so that corneocyte surfaces that were pointing towards the body inside were prepaired for the SEM scan. Another part of the tape with the adhering corneocytes was brought in contact with the Leit-Tab tape in such a manner that some less squamous epithelium cells, e.g. part of the cell ensemble, became adhered to the Leit-Tab tape. Consequently, the corneocyte surface pointing towards the body outside became nonattached and was observable with the electron microscope. The samples were gold-sputtered for 120s with a current of $40 \mathrm{~mA}$ (SCD050 Balzers, Liechtenstein) and subsequently studied using a scanning electron microscope (440i Leo, Oberkochen, Germany).

\section{Results}

The investigated corneocytes show an ordered three-dimensional architecture. The corneocyte outer surface (pointing towards the body outside) is different from the inner surface (towards inside) for both skin categories (Figure 1). It is obvious that the outside corneocyte surface is rather smooth, aside from some indentations.

Homogeneously distributed over the inner corneocyte surface, one finds small nubs in contrast to the outer surface arrangement. These nubs, described already by Menton and Eisen [9], show a unique appearance, similar to microvillis. Normally, the nubs height is not taller than their width. For the squamous epithelium cells selected, the base area of a representatively selected nub is about $600 \times 200 \mathrm{~nm}^{2}$. The negative indentations on the apical side show similar dimensions, e.g., $475 \times 275 \mathrm{~nm}^{2}$. Exemplary corneocyte thickness was determined for four different, half-skewed cells, providing a mean value including a standard deviation of $0.8 \mu \mathrm{m} \pm 0.2 \mu \mathrm{m}$.

A representative squamous epithelium cell of thin skin is shown in Figures 1a and c. The polyhedron of the basal cell can be recognized from its outer contour. However, the latter shape is no longer found in the corneocytes of thick skin samples.

\section{Morphology differences between corneocytes from thick and thin skin}

The tape stripping technique has the benefit to generate part of the fingerprint image. Therefore, it is possible to attribute most of shown corneocytes and corneocyte ensembles to a position at the fingertip. Here, the differences between the corneocytes structure in the skin recess are shown. The base area and shape of the corneocytes of thick skin varies according to the position in a ridge or fold of the skin recess. In general, these corneocytes possess a larger thickness than those obtained from thin skin samples. The architecture of these cells is also more complex. The outer surface of corneocytes from the heel of hand is rather flat, but showing a micro-structure (Figure 2). Many almost vertical indentations show similarities with a tyre profile (Figure $2 b$ ). In

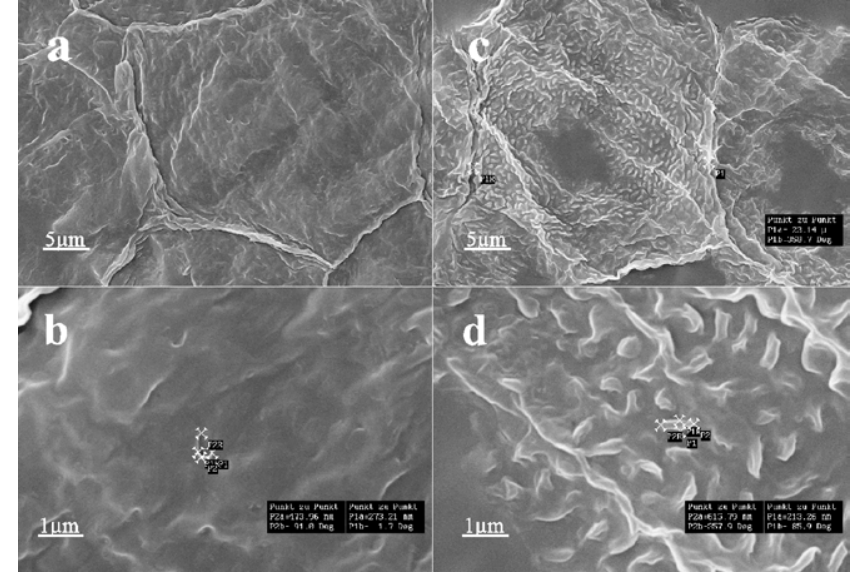

Figure 1: Representative corneocytes showing the different surfaces of thin skin. a) Surface towards the body outside: at the edges of the corneocytes one can clearly notice that the cells are layer-wise arranged. b) Enlargement of the cell outside surface; compared with the many nubs found on the inner cell surface, only a small number of indentations exists on the outer surface. c) Surface towards the body inside: Small ridges cross the cell from upper left to down right. On the top, bottom and in the middle of the photograph, the uniform black gluey surface of the tape can be identified; d) Enlargement of the inner surface part of the cell shown in (c). 
Citation: Stücker M, Licht M, Heise HM (2015) Surface Ultra-Structure and Size of Human Corneocytes from Upper Stratum Corneum Layers of Normal and Diabetic Subjects with Discussion of Cohesion Aspects. J Diabetes Metab 6: 603. doi:10.4172/2155-6156.1000603

contrast to this, the inner surface consists of a collection of small nubs with a height of about $1 \mu \mathrm{m}$, ordered densely and filigree-wise.

With their origin from thin skin, several corneoytes form an ensemble of a one-cell layer on the adhesive tape. On the other hand, given the complex architecture of the thick skin corneocytes, a uniform arrangement of cells cannot be determined for samples attached to the adhesive tape, as one or more cell layers are interwoven. The Figures 3 and 4 show a vis-à-vis of the outer and inner surfaces of such corneocytes.

\section{Morphology differences between thin skin corneocytes of healthy and diabetic subjects}

Concerning the skin ultrastructure, as found in thin skin of a diabetic patient, the nub structure on the inner corneocyte surface is showing a noticeably reduced contrast compared to skin samples from healthy subjects. The bottom side is covered by several ridges. The outer surface is still structured, but the indentations are less developed and lead to a softish appearance. In addition, the edges are frayed and tiny fragments can be found on the surface.

\section{Factors influencing mean corneocyte area}

As studied already in earlier publications, the corneocyte area has been found dependent on age or diabetic status. This has been traced back to differences in cell proliferation by Plewig [17], Kashibuchi et al. [18] and Sakai et al. [19]. In this study we also investigated two classes of subjects, i.e., healthy and diabetic subjects. For both categories we found an increase in the confidence interval of thin skin corneocytes after the age of seventy (Figure 5). When the individual classes were investigated separately, a relative augmentation of $14 \%$ of the average area of corneocytes from diabetic subjects as compared to the data obtained from healthy subjects can be stated (mean \pm SD area of corneocytes from healthy subjects: $886 \mu \mathrm{m}^{2} \pm 297 \mu \mathrm{m}^{2}$ and for diabetic

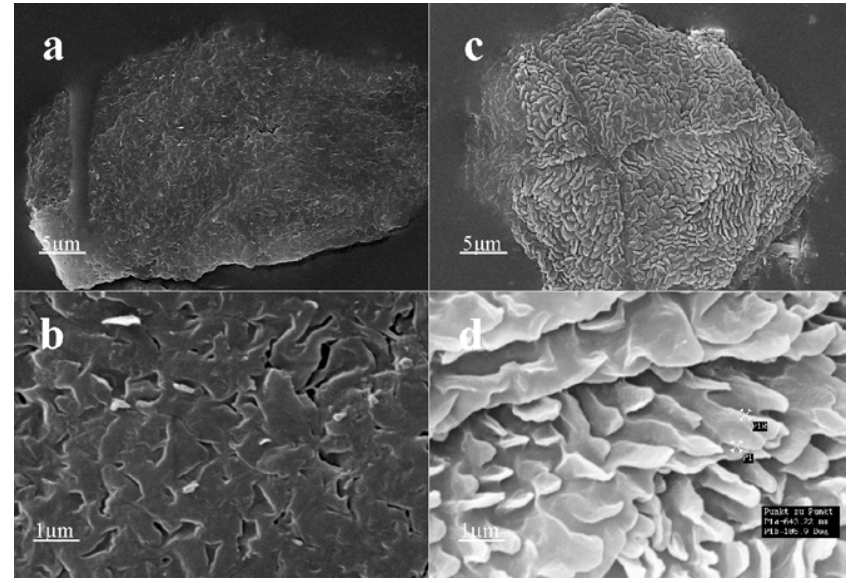

Figure 2: Representative corneocyte surfaces of thick skin (heel of hand) a) Surface towards the body outside. This corneocyte shows a smooth coverage with many irregular indentations on the whole outer surface. Many other corneocytes possess a smaller outside area than found for the inside surface. Smaller pale fragments can be identified as contamination on the surface (possibly from the interwoven, previously superimposed cell). The single cell is covered with pieces of glue from the tape, shown at the upper left to lower left side of the photo. b) Enlargement of the surface towards the body outside shown in (a). Here, the indentations show sharp edges. c) Surface towards the body inside of a single cell. Similar to a coral reef, the surface consists of many nobs. d) Enlargement of the inside surface shown in (c): Individual nobs possess a width of about $0.6 \mu \mathrm{m}$ at their outmost end.

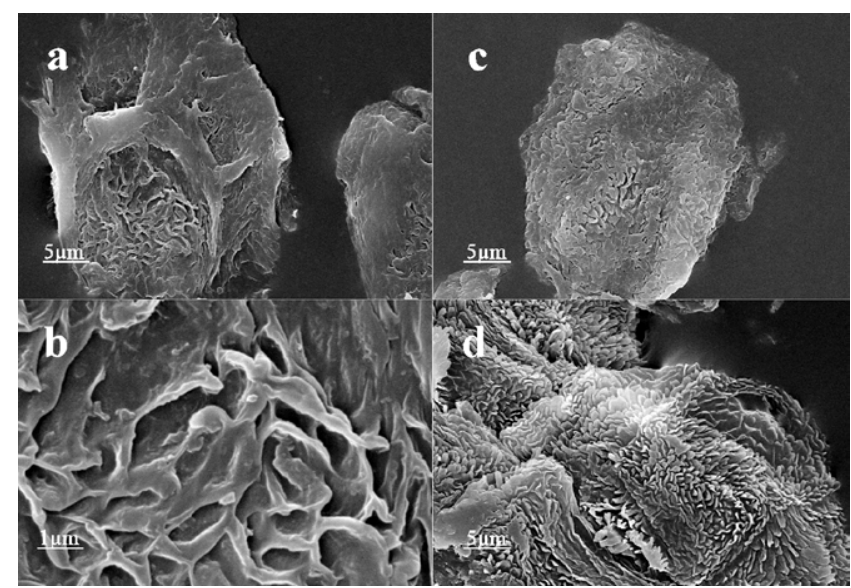

Figure 3: Representative corneocyte surfaces of thick skin (finger tip). a) Surface towards the body outside: large parts of the surface show indentations. b) Enlargement of the outside cell surface as shown in (a). In the middle of the photo a basketry-like structure is evident. c) Surface towards inside: The nubs have partially been flattened by the preparation. d) Another corneocytes surface towards body inside is viewed. The cell ensemble is extremely interwoven, so that the individual cell edges cannot be observed.



Figure 4: Examples of corneocyte surfaces of thin skin from a diabetic person. a) and b) Surface towards the body outside: Parts of the corneocytes show small fragmentation at the cell edges. c) and d) Surface towards the body inside: The nubs show less contrast than found for corneocytes from healthy persons.

subjects: $\left.1007 \mu \mathrm{m}^{2} \pm 377 \mu \mathrm{m}^{2}\right)$. However, this difference between the mean areas was found not to be significant $(\mathrm{P}>0.05)$.

\section{Discussion and Conclusion}

As discussed above, desquamation is influenced by several factors. In this context, the mechanical stability between corneocytes of the upper horny layer has been studied, which may be influenced by an interdigitation due to ultrastructural features found at both cell surfaces. At first it should be pointed out that the cells prepared by sputtering have been dehydrated in the vacuum. By the roughness depth of the thin skin corneocytes studied, it is realized that dehydration does not bring about a large morphological change with regard to cells that have been kept dry at normal atmosphere. The thickness of such thin skin corneocytes, according to measurements by Richter et al. [20], has been 
Citation: Stücker M, Licht M, Heise HM (2015) Surface Ultra-Structure and Size of Human Corneocytes from Upper Stratum Corneum Layers of Normal and Diabetic Subjects with Discussion of Cohesion Aspects. J Diabetes Metab 6: 603. doi:10.4172/2155-6156.1000603

Page 4 of 5

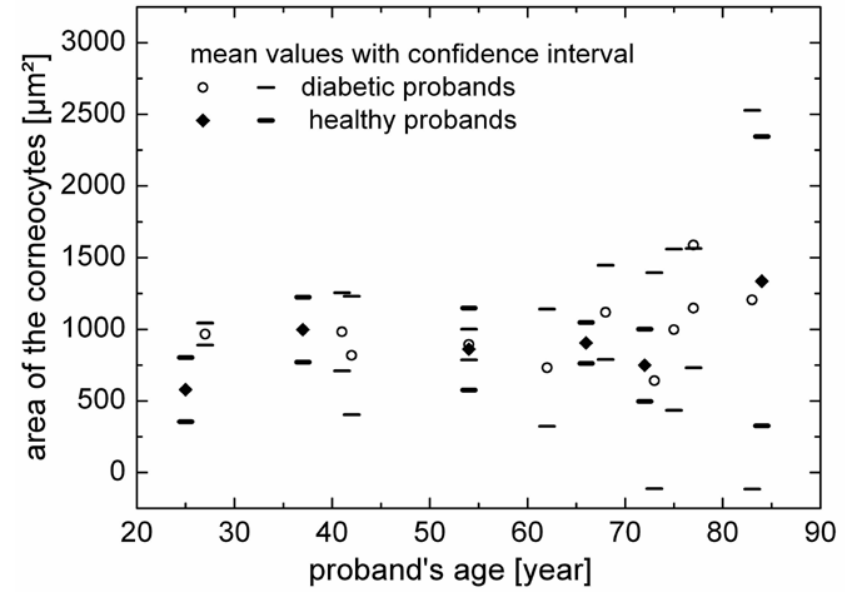

Figure 5: Mean corneocytes' area with dependency of subject age. Besides the mean area of corneocytes of thin skin, their $95 \%$ confidence intervals are also given.

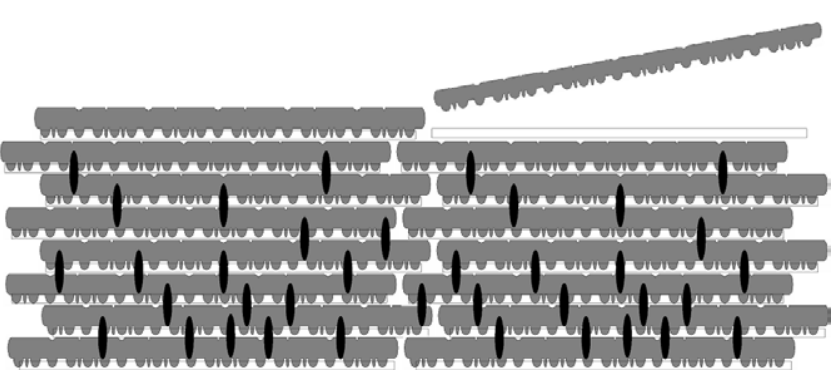

Figure 6: Extended skin model for the stratum corneum. Desmosomes are presented as black ellipses between the individual corneocytes furnished with a distinguished ultrastructure (shown as grey "bricks"). The lipid "mortar" substances are shown as light gray layers.

reported with values between $208 \mathrm{~nm}$ and $349 \mathrm{~nm}$ at normal humidity; this is significantly below the values that have been obtained by our measurements of the dehydrated and half-tilted corneocytes which allowed a thickness determination.

The ultrastructural phenomena as observed in thin skin of diabetic subjects can be described by slight changes in the way that the "nubs and indentation "structure is less developed and the surface appears to be more softish and frayed at the cellular edges than for corneocytes of healthy subjects. More studies have to be carried out for verifying this observation. For cell cultures of keratinocytes cultivated at elevated glucose concentrations it was observed that the keratinocytes became flattened and lost part of their orientation towards each other, furthermore, their proliferation was slowed down and an increased cell area was found by Spravchikov et al. [21].

For the human stratum corneum, the corneocyte area is certainly also dependent of the in-vivo cellular proliferation rate as evident under the presence of the skin disease of Lichen ruber planus. Under such conditions, the corneocytes, for which a reduced proliferation rate can be manifested, grow significantly larger; Plewig et al. [10]. On the other hand, in skin diseases such as Psoriasis vulgaris, for which an increase in proliferation rate can be found, a smaller corneocyte area on average is the consequence. Yajima et al. [22] found a significant correlation between corneocyte surface area and chronological age intervals in non-diabetic subjects $(\mathrm{N}=7)$, whereas there was no significant correlation in diabetic patients $(\mathrm{N}=7)$ with the $4^{\text {th }}, 7^{\text {th }}$ and $8^{\text {th }}$ decades of life. We also found insignificant differences of cell areas between non-diabetic and diabetic subjects. Our data show an age-dependent increase in cell area up to the $7^{\text {th }}$ decade of age. According to studies by Marks [23] and Plewig [17], the cell area has been claimed to be linearly dependent on age. The observed distribution of corneocyte areas may be the result of a combined effect from ageing and disease for diabetic subjects. In addition to the mean values, also an increase in variance can be observed based on the data set studied.

Responsible factors for ageing are certainly genetic, immune and "wear and tear" effects combined with repair-alteration. Glycation, cross-linking, oxidation and other chemical modifications all act to impair the molecular functioning of vital components such as DNA and structural proteins. Glycation of proteins contributes strongly too many progressive diseases, including complications from diabetes. Changes due to the latter disease have been monitored in conjunctive tissues, as manifested also by skin ageing.

Coming back to the corneocyte surface morphology, several positive aspects probably result from the differences in the surface ultrastructures. By the rather smooth external surface of our skin, the dynamic friction may be reduced and the evaporation surface for water vapour is essentially smaller than for a surface with increased roughness (Figure 6).

Different SC models have been discussed and illustrated in the literature. According to the "brick and mortar "model, lipids have an important role for the skin barrier formation and its stability. Here, lipid bilayers are glueing the different corneocyte bricks as described by Forslind [11]. An extension has been recently presented by Bernard et al. [4], detailing the corneosomal cohesion model. An important function is played by enzymes allowing a precisely controlled desquamation process. For the latter model, the corneocytes are still connected by desmosomes, which increase in numbers along a larger stratum corneum depth. Despite the identical cholesterol sulfate concentration in the SC of thick skin within the palm and the thin skin, e.g., of the upper arm, a seven-fold larger stability is found for the skin type mentioned first; Serizawa et al. [24]. Another fact is that for the thick skin, $50 \%$ of the intercellular gaps are equipped with desmosomes, whereas only a coverage of $20 \%$ is found for thin skin corneocytes as pointed out by Sheu and Tsai [25]. The much larger strength and cohesiveness of thick skin cannot be explained by the two factors only as discussed above. Our hypothesis is that additional interdigitation through the "nubs and indentation" ultrastructure of the corneocytes leads to the improved mechanical barrier characteristics observed for thick skin (Figure 6). Similar studies as presented by Wu et al. [26] must be carried out to quantify the intercellular delamination energy for the horny cells from different skin types as studied by us.

\section{Acknowledgements}

We would like to thank Mrs. Anne Szittnick for the support in the SEM measurements. Financial support by the Ministerium für Innovation, Wissenschaft Forschung und Technologie des Landes NRW and the Bundesministerium für Bildung und Forschung (BMBF, German Federal Ministry for Education and Research with project funds: FKZ 01EZ0203) is also gratefully acknowledged.

\section{References}

1. Brysk MM, Rajaraman S (1992) Cohesion and desquamation of epidermal stratum corneum. Prog Histochem Cytochem 25: 1-53.

2. Chapman SJ, Walsh A, Jackson SM, Friedmann PS (1991) Lipids, proteins and corneocyte adhesion. Arch Dermatol Res 283: 167-173.

3. Suzuki Y, Nomura J, Koyama J, Horii I (1994) The role of proteases in stratum 
Citation: Stücker M, Licht M, Heise HM (2015) Surface Ultra-Structure and Size of Human Corneocytes from Upper Stratum Corneum Layers of Normal and Diabetic Subjects with Discussion of Cohesion Aspects. J Diabetes Metab 6: 603. doi:10.4172/2155-6156.1000603

Page 5 of 5

corneum: involvement in stratum corneum desquamation. Arch Dermatol Res 286: $249-253$

4. Bernard D, Méhul B, Schmidt R (2002) Update on desquamation and first evidence for the presence of the endoglycosidase heparanase 1 in the human stratum corneum. In: Marks R, Lévêque JL, Voegeli R. The Essential Stratum Corneum. London: Martin Dunitz, p. 24.

5. Harding CR (2004) The stratum corneum: structure and function in health and disease. Dermatol Ther 17: 6-15.

6. Haftek M (2002) Ultrastructural aspects of the stratum corneum, In: Marks R, Lévêque JL, Voegeli R. The Essential Stratum Corneum. London: Martin Dunitz, p. 3-16.

7. Corcuff $P$, Fiat $F$, Minondo AM (2001) Ultrastructure of the human stratum corneum. Skin Pharmacol Appl Skin Physiol 14 Suppl 1: 4-9.

8. López O, Walther P, Cócera M, de la Maza A, Coderch L, et al. (2000) Structural modifications in the stratum corneum by effect of different solubilizing agents: a study based on high-resolution low-temperature scanning electron microscopy. Skin Pharmacol Appl Skin Physiol 13: 265-272.

9. Menton DN, Eisen AZ (1971) Structure and organization of mammalian stratum corneum. J Ultrastruct Res 35: 247-264.

10. Plewig G, Jansen T, Schürer NY (1997) [Stratum corneum]. Hautarzt 48: 510 521.

11. Forslind B (2000) Handbook of Occupational Dermatology. Berlin, Heidelberg, New York: Springer.

12. Warner RR, Stone KJ, Boissy YL (2003) Hydration disrupts human stratum corneum ultrastructure. J Invest Dermatol 120: 275-284.

13. Marks R (2004) The stratum corneum barrier: the final frontier. J Nutr 134 2017S-2021S.

14. Lindemann U, Wilken K, Weigmann HJ, Schaefer H, Sterry W, et al. (2003) Quantification of the horny layer using tape stripping and microscopic techniques. J Biomed Opt 8: 601-607.

15. Weigmann HJ, Lademann J, von Pelchrzim R, Sterry W, Hagemeister $T$, et al. (1999) Bioavailability of clobetasol propionate - Quantification of drug concentrations in the stratum corneum by dermatopharmacokinetics using tape stripping. Skin Pharmacol Appl Physiol 12: 46-53.
16. Weigmann HJ, Lademann J, Schanzer S, Lindemann U, von Pelchrzim R, et al. (2001) Correlation of the local distribution of topically applied substances inside the stratum corneum determined by tape-stripping to differences in bioavailability. Skin Pharmacol Appl Skin Physiol 14: 98-102.

17. Plewig G (1970) Regional differences of cell sizes in the human stratum corneum. II. Effects of sex and age. J Invest Dermatol 54: 19-23.

18. Kashibuchi N, Hirai Y, O'Goshi K, Tagami H (2002) Three-dimensional analyses of individual corneocytes with atomic force microscope: morphological changes related to age, location and to pathologic skin conditions. Skin Res Technol 8 : 203-211.

19. Sakai S, Endo Y, Ozawa N, Sugawara T, Kusaka A, et al. (2003) Characteristics of the epidermis and stratum corneum of hairless mice with experimentally induced diabetes mellitus. J Invest Dermatol 120: 79-85.

20. Richter T, Müller JH, Schwarz UD. Wepf R, Wiesendanger R (2001) Investigation of the swelling of human skin cells in liquid media by tapping mode scanning force microscopy. Appl Phys 72: 125-128.

21. Spravchikov N, Sizyakov G, Gartsbein M, Accili D, Tennenbaum T, et al. (2001) Glucose effects on skin keratinocytes: implications for diabetes skin complications. Diabetes 50: 1627-1635.

22. Yajima Y, Sueki H, Fujisawa R (1991) [Increased corneocyte surface area in the diabetic skin]. Nihon Hifuka Gakkai Zasshi 101: 129-134.

23. Marks $\mathrm{R}$ (1980) Epidermal and corneocyte size change with age. Br J Dermatol 102: 738-739.

24. Serizawa S, Osawa K, Tagshi K, Yamamoto A, Ito M (1992) Relationship between cholesterol sulfate and intercellular cohesion of the stratum corneum Demonstration using a push-pullmeter and an improved high performance thinlayer chromatographic separation. J Invest Dermatol 99: 232-236.

25. Sheu HM, Tsai JC (2002) Organization of the intercellular spaces of human palmo-plantar stratum corneum; in: Marks R, Lévêque JL, and Voegeli R: The Essential Stratum Corneum, London: Martin Dunitz, p. 89-91.

26. Wu KS, van Osdol WW, Dauskardt RH (2006) Mechanical properties of human stratum corneum: effects of temperature, hydration, and chemical treatment. Biomaterials 27: 785-795. 